\title{
Reaction Wheel Parameter Identification and Control through Receding Horizon-Based Null Motion Excitation
}

\author{
Avishai Weiss, Frederick Leve, Ilya Kolmanovsky, and Moriba Jah
}

\begin{abstract}
A torque tracking and parameter identification scheme using a receding horizon optimization is developed for an over-actuated spacecraft Reaction Wheel Array (RWA). Additional actuator motion, constrained to the null-space of the array, is provided in order to enhance persistence of excitation. Therefore, the system parameters are learned without inducing large perturbations to the controllable body (e.g., spacecraft bus). Constraining the excitation inputs to the nullspace, or null-motion excitation (NME), provides a way of adding information about the actuator misalignments with perturbations that are local to the trajectory, and scaled by the size of the parameter error. The discussed method provides a solution to the torque tracking problem, while simultaneously providing parameter identification through minimization of an appropriately defined objective function that penalizes the predicted parameter error covariance. The potential of the receding horizon approach to outperform the baseline null motion excitation algorithm proposed in earlier publications is demonstrated through simulations.
\end{abstract}

\section{INTRODUCTION}

This paper seeks to reduce the assembly, integration, and test (AI\&T) associated with detailed ground-based system identification of spacecraft through on-orbit estimation of actuator alignments. Due to a possible loss of communication, or other operational constraints, it may not be possible to apply an arbitrary tumble to a satellite for system identification. In these situations, the conditions required by many existing adaptive control and estimation techniques (see [1], [2], [3], [4], [5], [6], [7], [8], [9], [10] and references therein) to achieve both asymptotic tracking and asymptotic parameter identification may not be satisfied.

An approach to enhance on-board parameter identification via null motion excitation (NME) has been proposed in our previous work [11]. In it, an overactuated spacecraft is considered, and it is shown that the spacecraft actuators can be coordinated in such a way that the parameter estimate convergence is enhanced, while the disturbance to the nominal spacecraft attitude maneuver is minimized. In other words, it is demonstrated that information about parameters can be gained by adding NME to a nominal forced trajectory relative to the case of the forced trajectory by itself (i.e., passive

Graduate Student, Aerospace Engineering, University of Michigan, Ann Arbor, MI, 48109, avishai@umich.edu

Research Aerospace Engineer, Air Force Research Laboratory, Space Vehicles Directorate, Kirtland Air Force Base, NM, AFRL.RVSV@kirtland.af.mil

Professor, Aerospace Engineering, University of Michigan, Ann Arbor, MI, 48109, Ilya@umich.edu

Senior Engineer, Air Force Research Laboratory, Space Vehicles Directorate, Kirtland Air Force Base, NM, AFRL.RVSVQkirtland.af.mil system identification case). A local gradient approach was used in [11] to optimize the NME.

In this paper, a receding horizon optimization is proposed to provide the NME. In this approach, the NME sequence is optimized to minimize a functional of the predicted parameter error covariance over a finite time horizon, and the first element of the optimized sequence is applied to the spacecraft. The optimization is repeated at the next time instant using the updated error covariance matrix as an initial condition. The proposed approach is essentially an on-board design of experiments, used to enhance persistence of excitation conditions, and is related to our earlier work on receding horizon optimization for simultaneous tracking and parameter identification in automotive systems [12], [13]. The receding horizon approach of this paper is compared to the local gradient approach of [11], and it is shown that the potential for faster convergence exists at the price of higher computational cost. The differences between the receding horizon approach proposed in this paper and the local gradient method of [11] are that this approach considers the minimization of the total covariance (i.e., parameter and measurement) matrix and NME actuation " $n_{c}$ " steps ahead where the local gradient only considers minimizing the parameter error of perfect measurements one step ahead. As " $n_{c}$ " approaches the time of the maneuver the result will converge to the finite-time optimization of the chosen objective function. To the best of the authors' knowledge added persistence of excitation through the null-space alone or NME has only been published in [11] before this paper.

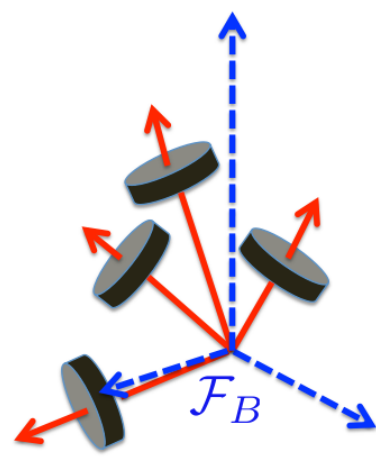

Fig. 1: Four Skewed RWA arrangement 


\section{SpaceCraft Dynamics Model}

We consider a rigid spacecraft bus actuated by a Reaction Wheel Array (RWA) consisting of four axially symmetric flywheels with negligible friction. See Figure 1. The total angular momentum of the spacecraft relative to its center of mass with respect to an inertial frame $\mathrm{F}_{\mathrm{E}}$ and resolved in a bus-fixed principal frame $\mathrm{F}_{\mathcal{B}}$ is given by

$$
\left.\vec{H}_{\mathrm{sc} / \mathrm{c} / \mathrm{E}}\right|_{\mathcal{B}}=J \omega+h,
$$

where $J$ is the spacecraft inertia matrix resolved in $\mathrm{F}_{\mathcal{B}}, \omega$ is the angular velocity of $F_{\mathcal{B}}$ with respect to $F_{E}$, resolved in $\mathrm{F}_{\mathcal{B}}$, and $h$ is the angular momentum of the RWA resolved in $\mathrm{F}_{\mathcal{B}}$. We assume zero external torque, and thus, the total angular momentum is conserved.

The inertial time derivative of (1) yields

$$
{\stackrel{\mathrm{E} \bullet}{H_{\mathrm{sc} / \mathrm{c} / \mathrm{E}}}}_{\mid \mathcal{B}}=J \dot{\omega}+\omega^{\times} J \omega+\dot{h}+\omega^{\times} h=0,
$$

where $\omega^{\times}$is the skew-symmetric matrix representing the cross-product, and

$$
\dot{h}=\frac{\partial h}{\partial \nu} \dot{\nu}=J_{\alpha}(\theta) \dot{\nu},
$$

where $J_{\alpha}(\theta)$ is the Jacobian matrix, which is a function of actuator alignments parameterized by a vector $\theta$, and $\nu=$ $\left[\begin{array}{llll}\nu_{1} & \nu_{2} & \nu_{3} & \nu_{4}\end{array}\right]^{\mathrm{T}} \in \mathbf{R}^{4}$ is a column vector of four flywheel rates.

Following [11], we can re-parameterize $h$ is terms of components of RWA alignment unit vectors as

$$
h=Y_{1}(\nu) \theta,
$$

where

$$
Y_{1}=I_{w}\left[\begin{array}{llll}
\nu_{1} I_{3} & \nu_{2} I_{3} & \nu_{3} I_{3} & \nu_{4} I_{4}
\end{array}\right],
$$

$I_{3}$ denotes the $3 \times 3$ identity matrix, and where $\theta \in \mathbf{R}^{12}$ is the parameter vector to be identified.

The NME approach is based on augmenting an excitation signal $n(t) \in \mathbf{R}^{4}$ to the nominal RWA control signal, $D(t) \in$ $\mathbf{R}^{4}$, so that

$$
\dot{\nu}=D(t)-\Gamma(\hat{\theta}(t)) n(t),
$$

where

$$
\Gamma(\hat{\theta}(t))=\left(I_{4}-J_{\alpha}^{\sharp}(\hat{\theta}) J_{\alpha}(\hat{\theta})\right),
$$

$I_{4}$ denotes the $4 \times 4$ identity matrix, and $J_{\alpha}^{\sharp}$ is the pseudoinverse of $J_{\alpha}, J_{\alpha}(\hat{\theta}) J_{\alpha}^{\sharp}(\hat{\theta})=I_{4}$. Note that the implementation of (5) is based on estimated alignments, $\hat{\theta}$, with the motivation that if $\hat{\theta}=\theta$, then $\dot{\nu}=D(t)$ and the effects of NME signal are zeroed out. Thus the overactuation capability of a 4 flywheel RWA system can be used to enhance the parameter identifiability. The computation of the excitation signal $n(t)$ is discussed in the next section.

\section{RECEDING HORIZON OPTIMIZATION OF THE NULL MOTION EXCITATION}

A discrete-time receding horizon approach is used for the optimization of NME signal $n(t)$ in (5). In the subsequent development, we use the notation $a(t+k \mid t)$ to denote the predicted value of a variable $a$ at the discrete time instant $t+k$ when the prediction is made at the discrete time instant $t$. Using this notation, and based on (5), the discrete-time update equations for the flywheel rates have the form

$$
\begin{aligned}
\nu(t+k+1 \mid t)= & \nu(t+k \mid t)+D(t) \Delta T \\
& -\Gamma(\hat{\theta}(t)) n(t+k \mid t) \Delta T,
\end{aligned}
$$

where $\Delta T$ is the sampling period, $\hat{\theta}(t) \in \mathbf{R}^{12}$ is the vector of the estimated reaction wheel alignment parameters, and $n \in \mathbf{R}^{4}$ is the null motion excitation signal that we determine through the receding horizon optimization.

With the motivation of simplifying the optimization problem, and with the justification that the nominal control law and adaptation are sufficiently slow, we do not predict $D$ and the parameter estimate changes over the horizon, thereby assuming $D(t+k \mid t)=D(t)$ and that $\hat{\theta}(t+k \mid t)=\hat{\theta}(t)$. Thus, in our approach, the term

$$
\Gamma(\hat{\theta}(t))=\left(I-J_{\alpha}^{\sharp}(\hat{\theta}) J_{\alpha}(\hat{\theta})\right),
$$

remains constant over the prediction horizon.

The optimization of the NME sequence is performed over a receding horizon of length $n_{c}$ so that $n(t+k \mid t), k=$ $0,1, \cdots, n_{c}$ minimizes a cost functional of the form

$$
J=\sum_{k=0}^{n_{c}} \operatorname{trace}(P(t+k \mid t))+\rho \cdot n^{\mathrm{T}}(t+k \mid t) n(t+k \mid t) .
$$

In (7), $P$ denotes the parameter error covariance matrix and $\rho$ is a weight penalizing the size of NME. Once the sequence is computed, the first element of it, $n(t)=n(t \mid t)$ is applied as an excitation and the process is repeated at the next time instant, $t+1$.

By combining (1) and (4), one obtains a linear regression model for identifying the parameter vector $\theta$,

$$
\begin{gathered}
y=Y_{1}(\nu) \theta+\epsilon, \\
y=\left.\vec{H}_{\mathrm{sc} / \mathrm{c} / \mathrm{E}}\right|_{\mathcal{B}}-J \omega,
\end{gathered}
$$

where $\left.\vec{H}_{\mathrm{sc} / \mathrm{c} / \mathrm{E}}\right|_{\mathcal{B}}-J \omega$ represents the measurement, with the added measurement noise, $\epsilon$. The assumption of $y$ being a measured signal is reasonable given that $\omega$ is measured, $J$ is known, the spacecraft orientation is measured and the total angular momentum vector $\vec{H}_{\mathrm{sc} / \mathrm{c} / \mathrm{E}}$ is conserved and is known at the initial time ${ }^{1}$. It should be noted that because both noise processes are assumed to be Gaussian zero mean,

\footnotetext{
${ }^{1}$ In case $\vec{H}_{\mathrm{sc} / \mathrm{c} / \mathrm{E}}$ is unknown at the initial time, it can be estimated along with $\theta$ using the approach in this paper.
} 
the addition of Gaussian variables associated with the RWA encoder and gyro noises is also a Gaussian random variable. It is assumed that all flywheel and gyro biases are removed separately from the attitude determination system. It should also be noted that the addition of two measurements does not make use of the measurement covariance optimally in the Kalman filter (i.e., the difference in accuracy of the two different sets of measurements are not exploited). Differencing of the measurements rather than considering separately was done to reduce the computation of the measurement covariance from a $7 \times 7$ matrix to a $3 \times 3$.

To compute $P(t+k \mid t)$ in (7), we use Recursive Least Squares (RLS)-based prediction of the parameter error covariance matrix, based on the equations

$$
\begin{gathered}
K(t+k \mid t)=P(t+k-1 \mid t) Y_{1}(t+k \mid t)^{\mathrm{T}} \\
\times\left(Y_{1}(t+k \mid t) P(t+k-1 \mid t) Y_{1}(t+k \mid t)^{\mathrm{T}}+R\right)^{-1}, \\
P(t+k \mid t)=\left(I_{12}-K(t+k \mid t) Y_{1}(t+k \mid t)\right) P(t+k-1 \mid t) \\
\times\left(I_{12}-K(t+k \mid t) Y_{1}(t+k \mid t)\right)^{\mathrm{T}} \\
+K(t+k \mid t) R K(t+k \mid t)^{\mathrm{T}}
\end{gathered}
$$

where $I_{12}$ denotes the $12 \times 12$ identity matrix and $R=$ $E\left[\epsilon(k) \epsilon(k)^{\mathrm{T}}\right]$ is the measurement noise covariance matrix. Note that Joseph's form of the a posteriori error covariance matrix update is used due to its better numerical conditioning properties.

In [11] a gradient type algorithm is used to update the parameter estimates. Here, for consistency with the RLS approach, updates of the form

$$
\hat{\theta}(t+1)=\hat{\theta}(t)+K(t+1 \mid t)\left(y(t+1)-Y_{1}(\nu(t+1)) \hat{\theta}(t)\right),
$$

are employed to extract parameter estimates.

\section{Simulation Setup}

Simulations are now presented to demonstrate the improved performance of the new receding horizon solution for the NME. The four flywheel RWA to be simulated has a three-orthogonal plus one skew arrangement shown in Figure 1.

The spacecraft and simulated maneuver parameters are $J=\operatorname{diag}(10,20,60)\left[\mathrm{kg} \mathrm{m}{ }^{2}\right], I_{w}=0.001\left[\mathrm{~kg} \mathrm{~m}^{2}\right]$, $\nu(0)=\left[\begin{array}{llll}0 & 0 & 0 & 0\end{array}\right]^{\mathrm{T}}[\mathrm{rad} / \mathrm{sec}], \omega(0)=\left[\begin{array}{lll}0 & 0 & 0\end{array}\right]^{\mathrm{T}}[\mathrm{rad} / \mathrm{sec}]$,

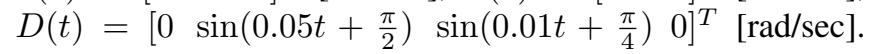
Note that based on the initial conditions of the simulation,
$\left.\vec{H}_{\mathrm{sc} / \mathrm{c} / \mathrm{E}}\right|_{\mathcal{B}}=0$. The initial parameter estimates are

$$
\hat{\theta}(0)=\left[\begin{array}{c}
0.7121 \\
0.0928 \\
0.6959 \\
0.0928 \\
0.7121 \\
0.6959 \\
0.6845 \\
0.7290 \\
0 \\
0.9916 \\
0.1292 \\
0
\end{array}\right],
$$

while the true RWA alignment parameters are

$$
\theta=\left[\begin{array}{c}
0.7037 \\
0.0693 \\
0.7071 \\
0.0693 \\
0.7037 \\
0.7071 \\
0.9952 \\
0.0980 \\
0 \\
0 \\
-0.7071 \\
0.7071
\end{array}\right]
$$

The initial parameter error covariance matrix is assumed to be of the form,

$$
P(0 \mid 0)=\frac{1}{3} I_{12},
$$

where $I_{12}$ is the $12 \times 12$ identity matrix. The covariance of the measurement noise $\epsilon$ in (8) has been estimated assuming $0.0005 \mathrm{rad} / \mathrm{sec}$ independent error standard deviations in measuring the components of the angular velocity vector $\omega$, and $2 \mathrm{rad} / \mathrm{sec}$ independent error standard deviations in measuring the components of $\nu$ so that

$$
R=10^{-3} \times \operatorname{diag}(0.0290,0.1040,0.9040) .
$$

\section{A. Case 1: Baseline Adaptation Algorithm with no NME}

The first case to be simulated is the baseline adaptation algorithm of reference [11] which is specified, in continuoustime as,

$$
\dot{\hat{\theta}}=\gamma Y_{1}(\nu)^{T}\left[-J \omega-Y_{1}(\nu) \hat{\theta}\right]
$$

and where we choose $\gamma=10 I_{12}$. In this case, there is no excitation in the null-space, and $n(t)=0$.

1) Results: The angular velocity of the spacecraft and RWA flywheel rates are shown in Figures 2 and 3. The parameter error, shown in Figure 4, does not converge to zero. This is because the forced trajectory followed by the spacecraft does not ensure persistency of excitation. The angular momentum error of the spacecraft-RWA array system in Figure 5 asymptotically approaches zero. 


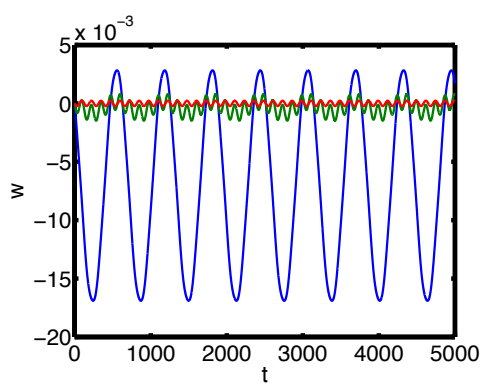

Fig. 2: The time histories of the spacecraft angular velocity components with an adaptation algorithm (11) and no NME.

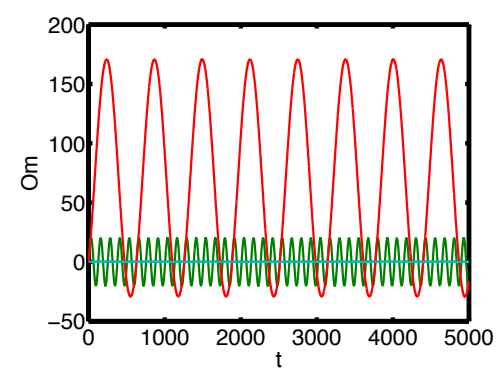

Fig. 3: The time histories of the RWA flywheel rates with an adaptation algorithm (11) and no NME.

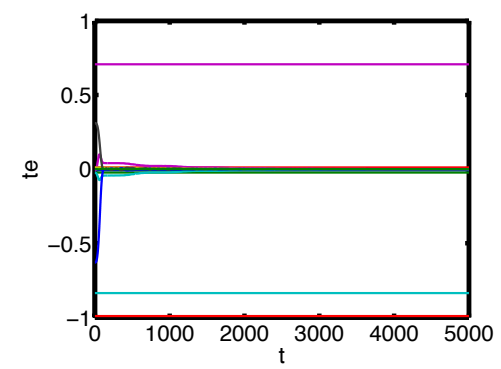

Fig. 4: The time histories of the parameter estimation errors with an adaptation algorithm (11) and no NME.

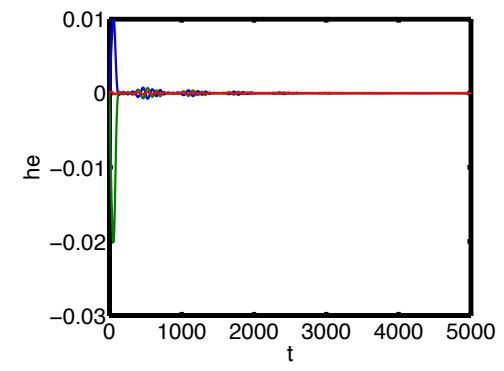

Fig. 5: The time history of the angular momentum error with an adaptation algorithm (11) and no NME.

\section{B. Case 2: Baseline Adaptation Algorithm and Local Gradient-Based NME Solution}

The local gradient-based NME signal from reference [11] is now augmented to enhance excitation and facilitate parameter identification. The parameters are estimated by
(11), and NME signal, $n(t)$, is generated in the direction of the gradient with respect to $\nu(t)$ of the objective function

$$
f=\sum_{i=1}^{3 n_{a}} \sum_{j=1}^{3 n_{a}} q_{i}^{\mathrm{T}}(t) q_{j}(t)
$$

where $n_{a}=4$ is the number of RWA actuators, and $q_{i}(t)$ and $q_{j}(t)$ are the ith and $j t h$ columns of the matrix

$$
Q(t)=\left(Y_{1}^{T}(t) Y_{1}(t)+\sum_{i=1}^{N_{b}-1} Y_{1}^{T}(t-i) Y_{1}(t-i)\right) .
$$

Here $N_{b}$ designates the past time window over which $Q$ is computed, and $Y_{1}(t)$ depends on $\nu(t)$. The NME from this algorithm adds excitation to the system along the trajectory thereby providing more information and making it possible to identify the parameters of the system without degrading commanded torque tracking performance. This approach's objective function differs from that of the proposed receding horizon approach in it has the same objective to maximize a form of information through the product of the regression matrices $Q(t)$, although, it does this in a locally optimal setting only one step ahead, considers perfect measurements, and does not minimize actuation.

1) Results: The angular velocity of the spacecraft and RWA flywheel rates are shown in Figures 6 and 7. The parameter error using this method is shown in Figure 8. Note that unlike the case without NME, the parameter error converges to zero, however, it has not converged sufficiently over the time interval of $5000 \mathrm{sec}$. The angular momentum error converges with oscillations at the steady state as shown in Figure 9. The flywheel actuation is shown in Figures 7 and the additional null motion added is shown in Figure 10. More details and discussion of the convergence of the parameters and the trajectory can be found in reference [11].

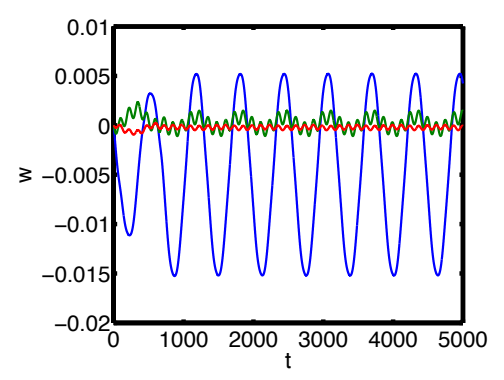

Fig. 6: The time histories of the spacecraft angular velocity components with the baseline NME algorithm and adaptation algorithm given by (11).

\section{Case 3: RLS Adaptation Algorithm and Receding Horizon NME Solution}

The final case in simulation is that of the NME solution found from a receding horizon optimization. It is expected that because the method of choosing NME here is local but based off a larger than a single time-step horizon and that the 


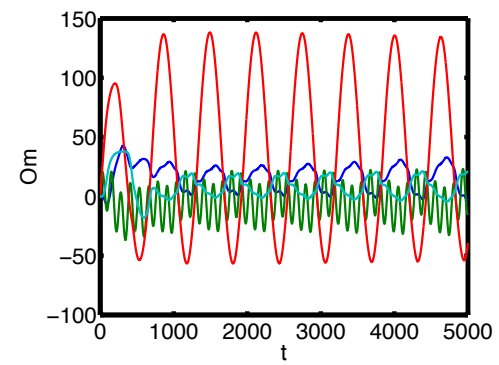

Fig. 7: The time histories of the RWA rates with the baseline NME algorithm and adaptation algorithm given by (11).

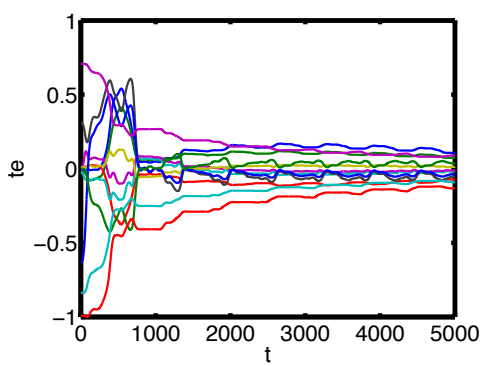

Fig. 8: The time histories of the parameter estimation errors with the baseline NME algorithm and adaptation algorithm given by (11).

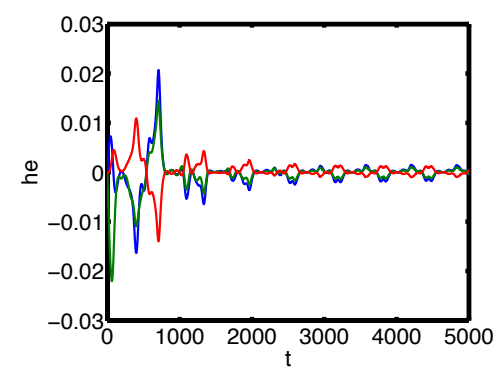

Fig. 9: The time history of the angular momentum error with the baseline NME algorithm and adaptation algorithm given by (11).

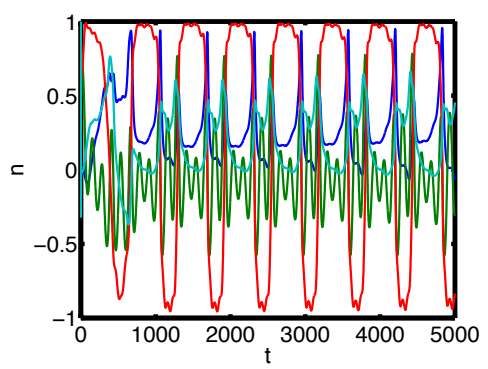

Fig. 10: The time history of the excitation signal $n$ with the baseline NME algorithm and adaptation algorithm given by (11).

objective function for the this method considers minimizing the NME actuation and covariance, that this method will perform superior to the local-gradient method in Section 4-
B. Note also that in the receding horizon case, the noise in the measurements is accounted for in the covariance prediction.

1) Results: To ensure that the excitation is maintained over time, the weight $\rho$ in (7) is made time-varying and decreased at a linear rate to a constant value. See Figure 11. See also reference [13] for additional remarks. We set $\Delta T=$ $1 \mathrm{sec}$ and we use the horizon $n_{c}=10$ in (7). While shorter horizons can reduce the computational time and effort, for the assumed levels of measurement noise shorter horizons produce slower parameter error convergence.

The angular velocity of the spacecraft and RWA flywheel rates are shown in Figures 12 and 13. The parameter error for the receding horizon method of NME exhibits faster parameter error convergence over $5000 \mathrm{sec}$ time interval versus the local gradient approach, compare Figures 14 and 8. In addition, the angular momentum error is smaller than with the local-gradient method, compare Figures 15 and 9. Finally, the additional actuation is an order of magnitude less with the receding horizon approach than with the local gradient method which is evident by comparing the null motion added in Figure 16 and Figure 10. Figure 17 demonstrates that the error covariance matrix is decreasing through plotting of the maximum $1-\sigma$ bounds on the covariance matrix. We emphasize that these results are not due to the difference between the parameter update laws (10) and (11); For instance, by setting $n(t)=0$ and executing (10), the parameter estimates do not converge to zero, as shown in Figure 18.

Even though the results are dependent on the choices of each algorithm parameters, they do indicate that the receding horizon approach has a potential to induce null motion excitation that facilitates fast parameter adaptation and smaller perturbations to the spacecraft albeit at a higher computational cost.

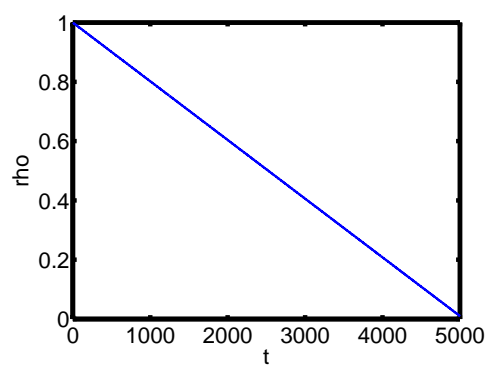

Fig. 11: The time history of the weight $\rho$.

\section{CONCLUSiON}

The Null Motion Excitation (NME) takes advantage of the spacecraft actuation redundancy (over-actuation) to provide excitation that enhances parameter estimation while minimizing the disturbance to the nominal spacecraft maneuver. A specific approach to NME is proposed in this paper. This approach is based on the receding horizon optimization of the 


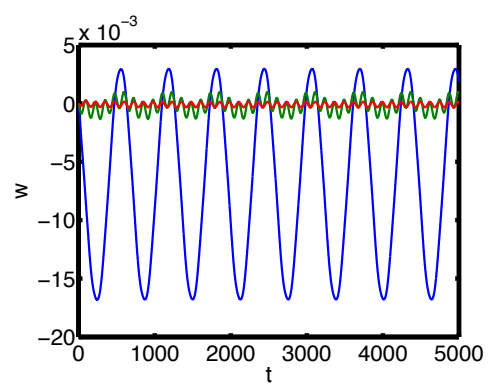

Fig. 12: The time histories of the spacecraft angular velocity components with the receding horizon NME algorithm.

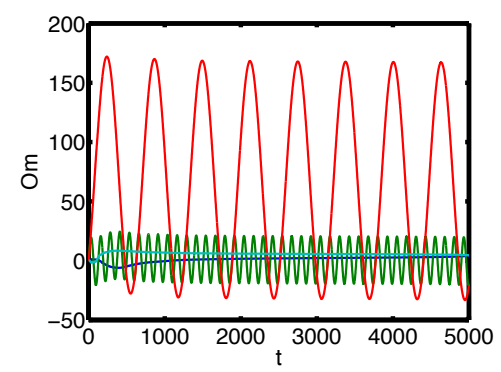

Fig. 13: The time histories of the RWA rates with the receding horizon NME algorithm.

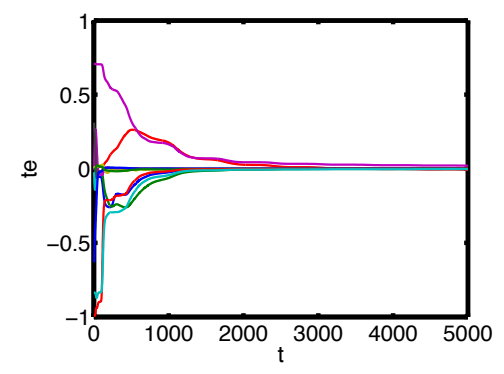

Fig. 14: The time histories of the parameter estimation errors with the receding horizon NME algorithm.

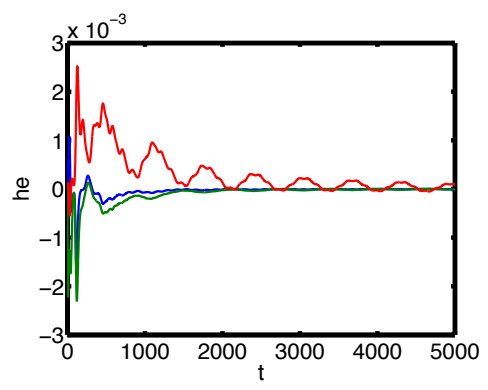

Fig. 15: The time history of the angular momentum error with the receding horizon NME algorithm.

excitation input to minimize the predicted estimation error covariance. Simulation results for the case of identifying alignments in the Reaction Wheel Assembly demonstrate that the receding horizon approach ensures faster parameter convergence versus zero excitation case, and that it has a potential to outperform a previously proposed algorithm in

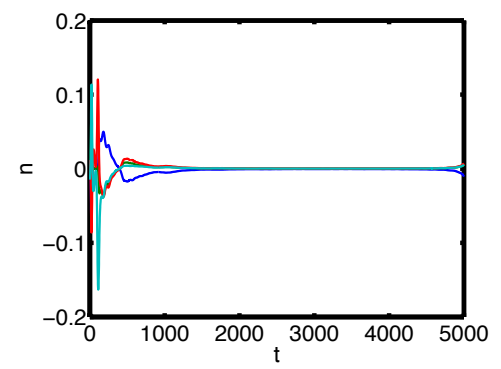

Fig. 16: The time history of the signal $n$ with the receding horizon NME algorithm.

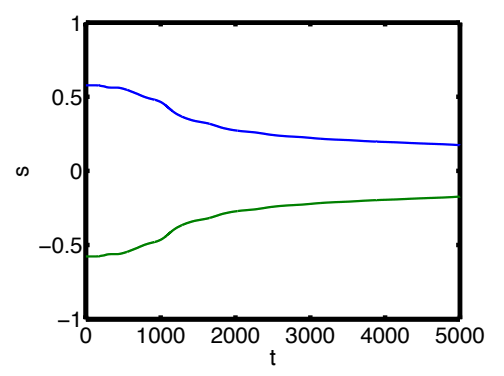

Fig. 17: The time history of the maximum 1- $\sigma$ standard deviation with the receding horizon NME algorithm.

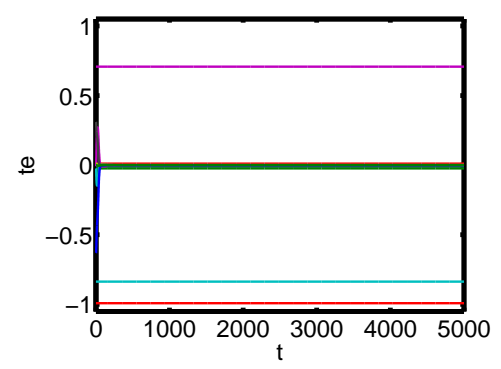

Fig. 18: The time histories of the parameter estimation errors with zero NME and parameter estimation based on (10).

[11] albeit at a higher on-board computational cost.

Several assumptions were made to simplify the treatment of the problem, that will be relaxed in the future publications. Various enhancements will be pursued. In particular an approach which uses a Gaussian sum in place of the predicted covariance for an objective function will be investigated. This is needed for some sensors such as electro-optical sensors for which the measurement noise has a Poisson rather than a Gaussian distribution. Other future work will be applying this type of solution incorporating NME to a slew of different problems (e.g., CMG gimbal axis alignments).

\section{ACKNOWLEDGEMENTS}

The authors gratefully acknowledge the contribution from the Air Force Office of Scientific Research under the lab task (LRIR11RV15COR) to support this research. 


\section{REFERENCES}

[1] W. MacKunis, K. Dupree, N. Fitz-Coy, and W. Dixon, "Adaptive satellite attitude control in the presence of inertia and CMG gimbal friction uncertainties," Journal of the Astronautical Sciences, vol. 56, no. 1, pp. 121-134, 2008.

[2] W. Dixon, Nonlinear control of engineering systems: A Lyapunovbased approach. Birkhauser, 2003.

[3] T. Mercker and M. Akella, "Onboard adaptive compensation for largescale misalignments in responsive space systems," in AAS Guidance and Control Conference, Breckenridge, Co, 2010.

[4] J. Ahmed, V. Coppola, and D. Bernstein, "Asymptotic tracking of spacecraft attitude motion with inertia matrix identification," in $D e$ cision and Control, 1997., Proceedings of the 36th IEEE Conference on, vol. 3. IEEE, 2002, pp. 2471-2476.

[5] N. Chaturvedi, D. Bernstein, J. Ahmed, F. Bacconi, and N. McClamroch, "Globally convergent adaptive tracking of angular velocity and inertia identification for a 3-DOF rigid body," Control Systems Technology, IEEE Transactions on, vol. 14, no. 5, pp. 841-853, 2006.

[6] O. Ma, H. Dang, and K. Pham, "On-Orbit Identification of Inertia Properties of Spacecraft Using a Robotic Arm," Journal of guidance, control, and dynamics, vol. 31, no. 6, 2008.

[7] A. Chakrabortty, M. Arcak, and P. Tsiotras, "Robust design of a spacecraft attitude tracking control system with actuator uncertainties," in Decision and Control, 2008. CDC 2008. 47th IEEE Conference on. IEEE, 2009, pp. 1587-1592.

[8] B. Costic, D. Dawson, M. De Queiroz, and V. Kapila, "A quaternionbased adaptive attitude tracking controller without velocity measurements," in Decision and Control, 2000. Proceedings of the 39th IEEE Conference on, vol. 3. IEEE, 2002, pp. 2424-2429.

[9] K. Astrom and B. Wittenmark, Adaptive control. Addison-Wesley Longman Publishing Co., Inc. Boston, MA, USA, 1994.

[10] S. Sastry and M. Bodson, Adaptive control: stability, convergence, and robustness, 1989.

[11] F. Leve and M. Jah, "Spacecraft actuator alignment determination through null motion excitation," in Proceedings of 62nd International Astronautical Congress, 2011.

[12] I. Kolmanovsky and V. Winstead, "A receding horizon optimal control approach to active state and parameter estimation in automotive systems," in Proceedings of 2006 IEEE Conference on Control Applications. IEEE, 2006, pp. 2796-2801.

[13] I. Kolmanovsky and D. Filev, Identification for Automotive Systems: "Optimal finite and receding horizon control for identification in automotive systems". Springer, LNCIS, 2012, ch. 18, pp. 327-348. 